\title{
Correction to: Surface Warping Incorporating Machine Learning Assisted Domain Likelihood Estimation: A New Paradigm in Mine Geology Modeling and Automation
}

\author{
Raymond Leung $^{1}$ - Mehala Balamurali ${ }^{1}$. \\ Alexander Lowe $^{1}$ (D)
}

Published online: 11 November 2021

(C) International Association for Mathematical Geosciences 2021

\section{Correction to: Mathematical Geosciences https://doi.org/10.1007/s11004-021-09967-5}

The figure belonging to Table 3 was missing from this article; the figure should have appeared as shown below.

The original article can be found online at https://doi.org/10.1007/s11004-021-09967-5.

Raymond Leung

raymond.leung@sydney.edu.au

Mehala Balamurali

mehala.balamurali@sydney.edu.au

Alexander Lowe

alexander.lowe@sydney.edu.au

1 The Australian Centre for Field Robotics, The University of Sydney, Sydney Robotics Hub J18, Sydney, NSW 2006, Australia 
Table 3 Multi-class geozone classification recall rate among the top- $n$ most likely candidates

\begin{tabular}{lllllllll}
\hline Classifier & \multicolumn{7}{l}{ Top- $n$ recognition rate (\%) } \\
\cline { 2 - 8 } & $n=1$ & $n=2$ & $n=3$ & $n=4$ & $n=5$ & $n=6$ & $n=8$ & $n=10$ \\
\hline Logistic & 51.40 & 71.92 & 78.79 & 84.61 & 88.18 & 90.91 & 94.40 & 96.36 \\
GaussianNB & 51.78 & 72.18 & 80.89 & 86.03 & 89.52 & 91.87 & 94.71 & 96.45 \\
KNN & 53.45 & 71.99 & 78.98 & 82.33 & 84.39 & 85.78 & 88.17 & 90.36 \\
L-SVC & 57.45 & 76.41 & 85.18 & 89.75 & 92.48 & 94.19 & 96.51 & 97.89 \\
RBF-SVM & 53.95 & 74.31 & 82.54 & 87.51 & 90.61 & 92.86 & 95.73 & 97.29 \\
GradBoost & 64.11 & 81.58 & 88.43 & 92.18 & 94.29 & 95.60 & 97.14 & 97.99 \\
MLP & 66.30 & 83.59 & 90.11 & 93.67 & 95.52 & 96.71 & 98.07 & 98.80 \\
RandomForest & 68.98 & 85.56 & 91.55 & 94.60 & 96.15 & 97.09 & 98.22 & 98.81 \\
\hline
\end{tabular}

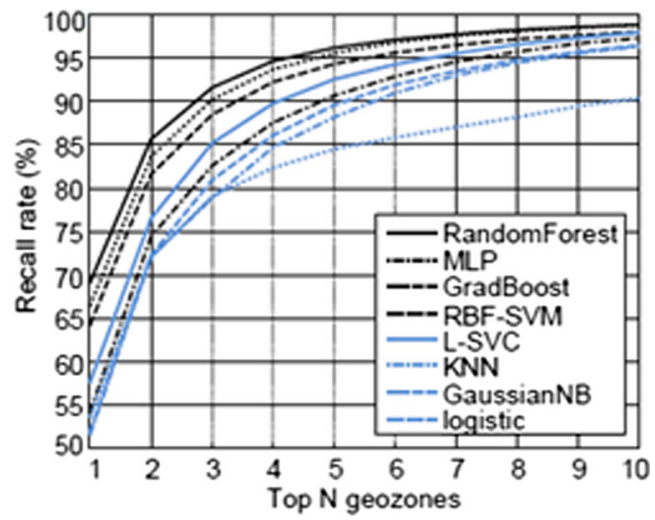

The original article has been corrected. We apologise for any inconvenience caused to our readers. 